\title{
Early Functional and Morphologic Vascular Renal Consequences of the Diabetic State
}

Dear Sir,

We read with interest the review article on Early Functional and Morphologic Vascular Renal Consequence of the Diabetic State (Diabetologia (1979) 17: 71-79) by Dr. Mogensen and his colleagues, but would like to comment in particular upon albumin excretion where the studies at Guy's Hospital have yielded a number of differences from those at Århus.

In contrast to Dr. Mogensen's findings we have shown [1] that mean urinary albumin excretion can be elevated under ordinary conditions of life and during conventional treatment in clinically non-proteinuric insulin-dependent diabetics with duration of disease ranging from 6 months to 39 years. This was also true for each group when the patients were divided into duration categories $0-10,11-20,21-39$ years. Interestingly enough the group with the longest duration of disease had the smallest proportion of patients with subclinically elevated albuminuria; we speculated that this could be due to the deliberate exclusion of patients with clinical proteinuria and/or premature mortality of proteinuric patients. We have extended these observations to another 30 insulin-dependent diabetics and confirmed our original findings.

In a recent publication [2] we have suggested that, although the evidence is based on prevalence studies, Mogensen's studies would support the view of an abrupt passage from normal excretion of albumin to clinically overt proteinuria, whereas our findings would support the view of a gradual increase of albumin excretion until the appearance of manifest albuminuria. This is an important difference in considering the natural history of diabetic renal disease and also in view of the fact that subclinically elevated albuminuria can be promptly reduced by optimal glycaemic control [1]. While the Århus workers [3] have shown a reduction in albumin excretion when insulin treatment is commenced in previously untreated patients and an increase in albumin excretion when insulin is withdrawn for some days, in our study [1] conventional blood glucose regulation was compared with optimal blood glucose control achieved by continuous subcutaneous insulin infusion (CSII). During CSII the total daily insulin dose remained unaltered in 3 patients, was reduced in 2 and was increased only in the other 2 in comparison with the daily insulin dose during conventional treatment. This suggests that our diabetics were not insufficiently treated during their conventional insulin regimen. We concluded that a change in the quality of glycaemic control can reduce the elevated capillary glomerular permeability to albumin, in that $\beta_{2}$-microglobulin excretion (an index of tubular function) was normal both during conventional and optimal blood glucose control.

Under stress situations (e.g. physical exercise) the urinary albumin excretion becomes exaggerated even in those patients who have normal albuminuria $[4,5]$. Patients with elevated resting albuminuria show higher mean absolute values after exercise, but we found [5] that the increment above baseline was not different from that of patients with normal resting albuminuria. Moreover, in our admittedly small series of 16 patients we did not find any correlation between exercise-induced albuminuria and duration of diabetes. The finding in our study [5] that the urinary excretion of $\beta_{2}$-microglobulin does not increase during exercise led us to conclude that the exaggerated exercise albuminuria is due to an increased glomerular permeability. This agrees with recent findings of Mogensen et al. [6] but is at variance with the findings of Poortmans et al. [7]. As to the effect of insulin on renal albumin excretion in a recent controlled study McKay and Viberti were unable to confirm Mogensen's data (Fig. 1). 


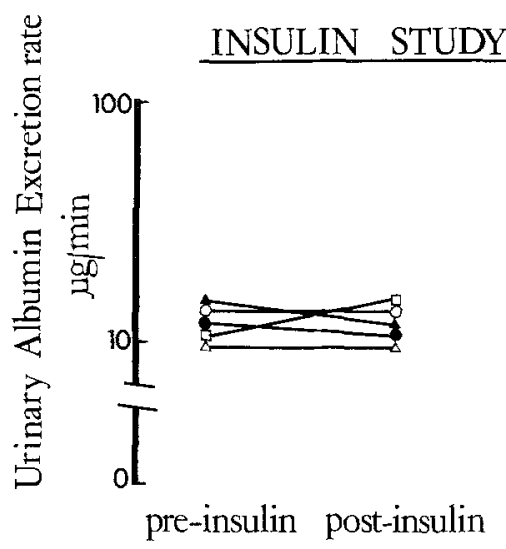

Finally, we would like to comment on the causes of the high GFR in diabetic patients. Mogensen's discussion of this (p. 72-73) contains a number of statements which must be questioned in the light of recent developments in the study of glomerular ultrafiltration dynamics. Although no direct data are available for man, filtration equilibrium (FE) has been shown to exist in the Munich-Wistar rat [8] and at least one primate species [9]. Filtration equilibrium is said to exist when ultrafiltration pressure (transcapillary hydraulic pressure gradient minus plasma oncotic pressure) falls to zero before the end of the glomerular capillary is reached, due to progressive haemoconcentration by abstraction of filtrate. Thus, filtration stops at some point along the length of the capillary. Since GFR is the arithmetical product of mean ultrafiltration pressure ( $\overline{\text { Puf }})$, glomerular surface area (S) and a term representing the conductivity of the membrane $(\mathrm{k})$, it is clear that under conditions of $\mathrm{FE}$ an increase in $\mathrm{S}$ or $\mathrm{k}$ or both cannot produce any further increase in GFR or filtration fraction (FF); this can only be achieved by an increase in Puf. Even if FE does not strictly obtain in the human, a large increase in $S$ would only lead to a small increment in GFR unless the degree of disequilibrium were very large, which is improbable. Furthermore, such a relationship would be highly non-linear, which is inconsistent with the finding that: 'a close correlation has been found between individual values of GFR and absolute filtration areas in short-term diabetics'.

How then to account for the observations of Mogensen et al.? The only hypothesis which adequately explains an increase in GFR and FF is that Puf is increased in the diabetic patient, and that this increase is proportionately greater than any associated increase in renal plasma flow. This requires an increase in efferent, and a decrease in afferent, arteriolar resistance. This will lead not only to increased $\overline{\mathrm{Puf}}$ but also to increased distending pressure. If the glomerular capillaries are compliant (as seems likely from their ultrastructure) an increase in capillary surface area would be expected, approximately proportional to the increase in pressure (and therefore to the increase in GFR).

The observation that GFR correlated with increased kidney size and weight [10] in diabetic patients is most interesting and perhaps throws further light on the nature of the process involved. The great bulk of kidney mass is tubules, glomeruli contributing but a small proportion of the total. We therefore infer that there is a proportionality between tubular mass and glomerular function in diabetes. There is growing evidence that glomerular haemodynamics are under the control of the tubule via a feedback loop operating at the point of approximation of the early distal tubule and the glomerular arterioles (macula densa and juxta-glomerular apparatus). Thus, control of GFR is mediated by changes in renal plasma flow (with proportionate changes in Puf); the glomeruli are normally operating far below their maximal functional capacity to the extent that GFR can be greatly elevated above normal values simply by increasing renal plasma flow no structural change is necessary.

We would, therefore, suggest the following scheme to account for the increased GFR of the diabetic patient:

1) Increased glucose delivery to the tubule leads to tubular hypertrophy in order to meet the increased need for glucose reabsorption.

2) Since glucose and sodium reabsorption in the proximal tubule are in part coupled, the obligate increase in sodium reabsorption accompanying the increase in glucose reabsorption requires that GFR increases to maintain glomerulotubular balance for salt and water - a prime requisite for survival.

3) The necessary increase in GFR is achieved, through tubulo-glomerular feedback, by an increase in efferent arteriolar resistance and a decrease in 
afferent arteriolar resistance leading to increased $\overline{\mathrm{Puf}}$ and an increase in GFR out of proportion to any increase in glomerular plasma flow, i. e. an increase in FF.

4) The increased distending pressure causes a secondary, passive increase in glomerular surface area which, for reasons given above, does not directly contribute to the increase in GFR and FF.

G. C. Viberti, M. D.

G. B. Haycock, M. B., M. R. C. P.

J. C. Pickup, B. M., D. Phil.

R. J. Jarrett, M. A., M. D.

H. Keen, M. D., F. R. C. P.

\section{References}

1. Viberti G C, Pickup J C, Jarrett R J, Keen H (1979) Effect of control of blood glucose on urinary excretion of albumin and $\beta_{2}$-microglobulin in insulin-dependent diabetes. $N$ Engl J Med 300: $638-641$

2. Viberti GC (1979) Early functional and morphological changes in diabetic nephropathy. Clin Nephrol 12: 47-53

3. Mogensen CE (1976) Renal function changes in diabetes. Diabetes 25: 872-79
4. Mogensen CE, Vittinghus E (1975) Urinary albumin excretion during exercise in juvenile diabetes. A provocation test for early abnormalities. Scand J Clin Lab Invest 35: 295-300

5. Viberti GC, Jarrett RJ, McCartney M, Keen H (1978) Increased glomerular permeability to albumin induced by exercise in diabetic subjects. Diabetologia 14: 293-300

6. Mogensen CE, Vittinghus E, Sølling K (in press) Abnormal albumin excretion after two provocative renal tests in diabetes: Physical exercise and lysine injection. Kidney Int

7. Poortmans J, Dewancker A, Dorchy H (1976) Urinary excretion of total protein, albumin and $\beta_{2}$-microglobulin during exercise in adolescent diabetics. Biomedicine 25: 273-274

8. Brenner BM, Troy J L, Daugherty T M, Deen WM, Robertson CR (1972) Dynamics of glomerular ultrafiltration in the rat. II. Plasma-flow dependence of GFR Am J Physiol 223 : $1184-1190$

9. Maddox D A, Deen WM, Brenner B M (1974) Dynamics of glomerular ultrafiltration. VI. Studies in the primate. Kidney Int 5: 271-278

10. Mogensen CE, Anderson MJF (1973) Increased kidney size and glomerular filtration rate in early juvenile onset diabetes. Diabetes 22: 706-712

G. C. Viberti, M. D.

Unit for Metabolic Medicine

Guy's Hospital Medical School

London SE1 9RT

England 